\title{
Spectral Analysis of Discontinuous Boundary-Value Problems with Retarded Argument
}

\author{
Erdoğan Şen
}

In the paper, we are concerned with spectral properties of discontinuous Sturm-Liouville type problems with retarded argument. We extend and generalize some approaches and results of the classical regular and discontinuous Sturm-Liouville problems. First, we study the spectral properties of a Sturm-Liouville problem on the half-axis and obtain lower bounds for the eigenvalues of this problem. Then we study spectral properties of a Sturm-Liouville problem with discontinuous weight function which contains a spectral parameter in the boundary conditions. We also obtain asymptotic formulas for eigenvalues and eigenfunctions of this problem and bounds for the distance between eigenvalues.

Key words: differential equation with retarded argument, eigenparameter, transmission conditions, asymptotics of eigenvalues, bounds for eigenvalues.

Mathematical Subject Classification 2010: 34L15, 34L20, 35R10

\section{Introduction}

The study of many physical phenomena, such as the vibration of strings, the interaction of atomic particles, the earth's free oscillations yields Sturm-Liouville problems. A Sturm-Liouville problem with eigenparameter contained in the boundary condition arises upon separation of variables in the one-dimensional wave and heat equations for a varied assortment of physical problems, e.g., in the diffusion of water vapour through a porous membrane and several electric circuit problems involving long cables. Differential equations with deviating argument, in particular differential equations with retarded argument, describe processes with after-effect. They have many applications in economics, biophysics, medicine, robotics, problems connected with combustion in rocket engines, etc.

Boundary-value problems with discontinuities inside the interval and regular boundary-value problems have been studied by many authors [1-24]. The asymptotic formulas for the eigenvalues and eigenfunctions of a boundary problem of

(C) Erdoğan Şen, 2018 
Sturm-Liouville type for the second order differential equation with retarded argument were obtained in $[3,4,16,17,19-21]$. In [17], S.B. Norkin considered the equation

$$
x^{\prime \prime}(t)+\lambda x(t)+M(t) x(t-\Delta(t))=0
$$

with boundary conditions

$$
\begin{aligned}
x(0) \cos \alpha+x^{\prime}(0) \sin \alpha & =0, \\
x(t-\Delta(t)) & =x(0) \phi(t-\Delta(t)) \quad \text { if } \quad t-\Delta(t)<0, \\
\sup _{[0, \infty)}|x(t)| & <\infty .
\end{aligned}
$$

Here $M(t)$ and $\Delta(t) \geq 0$ are defined and continuous on the half-axis $[0, \infty), \lambda$ is a real parameter $(-\infty<\lambda<+\infty), \alpha$ is an arbitrary real number, and $\phi(t)$ is a continuous initial function on the initial set $E_{0}$ with $\phi(0)=1$. S.B. Norkin proved the existence theorem and obtained lower bounds for eigenvalues of this problem. Motivated by [17], in this paper we study some spectral properties of eigenvalues and eigenfunctions of a discontinuous boundary value problem with retarded argument. Namely, we consider the boundary value problem for the differential equation

$$
u^{\prime \prime}(x)+M(x) u(x-\Delta(x))+\lambda u(x)=0
$$

on $[0, r) \cup(r, \infty)$ with boundary conditions

$$
\begin{aligned}
u(0) \cos \alpha+u^{\prime}(0) \sin \alpha & =0, \\
\sup |u(x)| & <\infty, \quad x \in[0, r) \cup(r, \infty), \\
u(x-\Delta(x)) & = \begin{cases}u(0) \phi_{1}(x-\Delta(x)) & \text { if } x-\Delta(x)<0, \\
u(r) \phi_{2}(x-\Delta(x)) & \text { if } x-\Delta(x)<r,\end{cases}
\end{aligned}
$$

and with transmission conditions

$$
\left\{\begin{aligned}
u(r-0) & =\delta u(r+0), \\
u^{\prime}(r-0) & =\delta u^{\prime}(r+0) .
\end{aligned}\right.
$$

Here the real-valued function $M(x)$ is continuous on $[0, r) \cup(r, \infty)$ and it has the finite limits

$$
\begin{aligned}
M(r \pm 0) & =\lim _{x \rightarrow r \pm 0} M(x) \\
\phi(x, \lambda) & = \begin{cases}\phi_{1}(x), & x \in E_{0}, \\
\phi_{2}(x), & x \in E_{1},\end{cases}
\end{aligned}
$$

is an initial function with $\phi_{1}(0)=1$ and $\phi_{2}(r)=1$; the real-valued function $\Delta(x) \geqslant 0$ is continuous on $[0, r) \cup(r, \infty)$ and it has the finite limits

$$
\Delta(r \pm 0)=\lim _{x \rightarrow r \pm 0} \Delta(x)
$$


$\lambda$ is a real parameter $(-\infty<\lambda<\infty)$, and $\alpha, r, \delta \neq 0(0<r<\infty)$ are arbitrary real numbers.

The paper is organized as follows: after the Introduction, we show that all positive values of the parameter $\lambda$ are eigenvalues of the problem (1.1)-(1.5) and then we prove the existence theorem for the problem (1.1)-(1.5). In the next section, we obtain lower bounds for the eigenvalues of the problem (1.1)-(1.5). In Section 4, the spectral properties of a Sturm-Liouville problem with discontinuous weight function which contains a spectral parameter in the boundary condition is studied. In Section 5, the asymptotic formulas for eigenvalues and eigenfinctions of the problem (4.1)-(4.5) are obtained. And in Section 6, which is the last section of the paper, we obtain bounds for the distance between the eigenvalues of the problem (4.1)-(4.5).

Let $w(x, \lambda)$ be as

$$
w(x, \lambda)= \begin{cases}w_{1}(x, \lambda), & x \in[0, r) \\ w_{2}(x, \lambda), & x \in(r, \infty)\end{cases}
$$

Here $w_{1}(x, \lambda)$ is a solution of equation $(1.1)$ on $[0, r)$ satisfying the initial conditions

$$
\left\{\begin{array}{l}
w_{1}(0, \lambda)=\sin \alpha, \quad w_{1}^{\prime}(0, \lambda)=-\cos \alpha, \\
w_{1}(x-\Delta(x), \lambda)=\sin \alpha \phi_{1}(x-\Delta(x)), \quad \text { if } x-\Delta(x)<0 .
\end{array}\right.
$$

After defining the above solution, we will define the solution $w_{2}(x, \lambda)$ of equation $(1.1)$ on $(r, \infty)$ by means of the solution $w_{1}(x, \lambda)$ by the initial conditions

$$
\left\{\begin{array}{l}
w_{2}(r, \lambda)=\delta^{-1} w_{1}(r, \lambda), \quad w_{2}^{\prime}(r, \lambda)=\delta^{-1} w_{1}^{\prime}(r, \lambda), \\
w_{2}(x-\Delta(x), \lambda)=\delta^{-1} w_{1}(r, \lambda) \phi_{2}(x-\Delta(x)), \quad \text { if } x-\Delta(x)<r .
\end{array}\right.
$$

Now we will state the following lemma which can be proved by using the method similar to that given in [3].

Lemma 1.1. Let $w(x, \lambda)$ be a solution of equation (1.1). Then the following integral equations hold:

$$
\begin{aligned}
w_{1}(x, \lambda)= & \sin \alpha \cos s x-\frac{\cos \alpha}{s} \sin s x \\
& -\frac{1}{s} \int_{0}^{x} M(\tau) \sin s(x-\tau) w_{1}(\tau-\Delta(\tau), \lambda) d \tau, \\
w_{1}(x, \lambda)= & \sin \alpha \cosh m x-\frac{\cos \alpha}{m} \sinh m x \quad \sqrt{\lambda}, \lambda>0, \\
& -\frac{1}{m} \int_{0}^{x} M(\tau) \sinh m(x-\tau) w_{1}(\tau-\Delta(\tau), \lambda) d \tau, \\
w_{2}(x, \lambda)= & \frac{1}{\delta} w_{1}(r, \lambda) \cos s(x-r)+\frac{w_{1}^{\prime}(r, \lambda)}{s \delta} \sin s(x-r)
\end{aligned}
$$




$$
\begin{aligned}
& -\frac{1}{s} \int_{r}^{x} M(\tau) \sin s(x-\tau) w_{2}(\tau-\Delta(\tau), \lambda) d \tau, \\
s=\sqrt{\lambda}, \lambda>0, & \\
w_{2}(x, \lambda)= & \frac{1}{\delta} w_{1}(r, \lambda) \cosh m(x-r)+\frac{w_{1}^{\prime}(r, \lambda)}{m \delta} \sinh m(x-r) \\
& -\frac{1}{m} \int_{r}^{x} M(\tau) \sinh m(x-\tau) w_{2}(\tau-\Delta(\tau), \lambda) d \tau, \\
w_{1}(x, 0)= & \sin \alpha-x \cos \alpha \\
& -\int_{0}^{x} M(\tau)(x-\tau) w_{1}(\tau-\Delta(\tau), \lambda) d \tau .
\end{aligned}
$$

\section{Existence theorems}

Theorem 2.1. Let

$$
\sup _{x \in E_{0} \cup E_{1}}|\phi(x)|=\phi_{0}<\infty
$$

and, in equation (1.1), let

$$
\int_{0}^{\infty}|M(\tau)| d \tau=M_{\infty}<\infty
$$

Then all positive values of the parameter $\lambda$ are eigenvalues of the boundary-value problem (1.1)-(1.5).

Proof. By (1.8), if $\lambda>0$, then

$$
w_{1}(x, \lambda)=R_{\lambda} \sin \left(s x-\psi_{\lambda}\right)-\frac{1}{s} \int_{0}^{x} M(\tau) \sin s(x-\tau) w_{1}(\tau-\Delta(\tau), \lambda) d \tau,
$$

where

$$
R_{\lambda}=\sqrt{\sin ^{2} \alpha+\frac{\cos ^{2} \alpha}{\lambda}}, \quad \cos \psi_{\lambda}=\frac{-\cos \alpha}{s R_{\lambda}}, \quad \sin \psi_{\lambda}=\frac{-\sin \alpha}{R_{\lambda}} \quad\left(0 \leq \psi_{\lambda}<2 \pi\right) .
$$

Let $x_{0} \in(0, r)$, and let $N_{\lambda}\left(x_{0}\right)=\max _{\left[0, x_{0}\right]}\left|w_{1}(x, \lambda)\right|$. In a similar way as in [17], for $s>0$, we obtain that

$$
N_{\lambda}\left(x_{0}\right) \leq \max \left\{R_{\lambda} \exp \frac{M_{\infty}}{s} ; R_{\lambda}+\frac{|\sin \alpha| \phi_{0} M_{\infty}}{s}\right\}<\infty .
$$

But the proof for $w_{2}(x, \lambda)$ needs a separate consideration. By (1.10), if $\lambda>0$, then

$$
\begin{aligned}
w_{2}(x, \lambda)= & Q_{\lambda} \sin \left(s(x-r)-\chi_{\lambda}\right) \\
& -\frac{1}{s} \int_{r}^{x} M(\tau) \sin s(x-\tau) w_{2}(\tau-\Delta(\tau), \lambda) d \tau,
\end{aligned}
$$


where

$$
\begin{gathered}
Q_{\lambda}=\sqrt{\delta^{-2} w_{1}^{2}(r, \lambda)+\frac{\delta^{-2}\left(w_{1}^{\prime}(r, \lambda)\right)^{2}}{\lambda}}, \\
\cos \chi_{\lambda}=\frac{\delta^{-1} w_{1}^{\prime}(r, \lambda)}{s Q_{\lambda}}, \quad \sin \chi_{\lambda}=\frac{-\delta^{-1} w_{1}(r, \lambda)}{Q_{\lambda}}, \quad 0 \leq \chi_{\lambda}<2 \pi .
\end{gathered}
$$

Let $x_{p} \in(r, \infty)$, and let $K_{\lambda}\left(x_{p}\right)=\max _{\left[r, x_{p}\right]}\left|w_{2}(x, \lambda)\right|$. Obviously, $K_{\lambda}\left(x_{p}\right) \geq$ $K_{\lambda}(x)\left(x_{p} \geq x\right)$ and from (1.7), (2.1)-(2.3), one of the following inequalities holds:

$$
K_{\lambda}\left(x_{p}\right) \leq Q_{\lambda}+\frac{1}{s} \int_{r}^{x_{p}}|M(\tau)| K_{\lambda}(\tau) d \tau
$$

or

$$
K_{\lambda}\left(x_{p}\right) \leq Q_{\lambda}+\frac{\left|\delta^{-1} w_{1}(r, \lambda)\right| \phi_{0}}{s} \int_{r}^{x_{p}}|M(\tau)| d \tau \leq Q_{\lambda}+\frac{\left|\delta^{-1} w_{1}(r, \lambda)\right| \phi_{0} M_{\infty}}{s} .
$$

By Lemma 2.3.5 from [17], it follows from (2.4) that

$$
K_{\lambda}\left(x_{p}\right) \leq Q_{\lambda} \exp \frac{1}{s} \int_{r}^{x_{p}}|M(\tau)| d \tau \leq Q_{\lambda} \exp \frac{M_{\infty}}{s}
$$

and for $s>0$,

$$
K_{\lambda}\left(x_{p}\right) \leq \max \left\{Q_{\lambda} \exp \frac{M_{\infty}}{s} ; Q_{\lambda}+\frac{\left|\delta^{-1} w_{1}(r, \lambda)\right| \phi_{0} M_{\infty}}{s}\right\}<\infty .
$$

The bound obtained is valid for any $\lambda>0$ and is independent of $x_{0}$, which proves the theorem.

Remark 2.2. Let $\sup _{[0, r) \cup(r, \infty)}|w(x, \lambda)|=\Omega_{\lambda}$. Then $\Omega_{\lambda}$ for $\lambda \geq \epsilon>0$ is bounded uniformly with respect to $\lambda$.

Lemma 2.3. In equation (1.1), let the functions $M(x)$ and $\Delta(x) \geq 0$ be defined and continuous on $[0, r) \cup(r, \infty)$,

$$
\begin{gathered}
x-\Delta(x) \geq A \quad(x \geq A), \\
\sup _{[A, r) \cup(r, \infty)}|M(x)|=M_{p}<\infty,
\end{gathered}
$$

and for $\lambda=s^{2}(s>0)$, let the number $\eta$ be such that

$$
\eta \sqrt{\lambda}>M_{p}
$$

Also let us define the solution of (1.1) as

$$
u(x)= \begin{cases}u_{1}(x), & x \in[0, r) \\ u_{2}(x), & x \in(r, \infty)\end{cases}
$$


Then the following bound holds for $u_{1}(x)$ and $u_{2}(x)$ and their derivatives:

$$
\begin{aligned}
\left|u_{j}^{(i)}(x)\right| \leq B_{j i} \exp (\eta(x-A)), i=0,1 ; & j=0,1 ; \\
& x \in\{x: A \leq x<\infty\} /\{r\},
\end{aligned}
$$

where $B_{i}$ are constants (to be defined in the proof).

Proof. The bounds for $u_{1}(x)$ and $u_{1}^{\prime}(x)$ can be obtained in the same way as in Lemma 4.2.1 from [17]. But the bounds for $u_{2}(x)$ and $u_{2}^{\prime}(x)$ need a separate consideration. Let $v(x)=u_{2}(x) \exp (-\eta x)$. Then, from (2.3.7) in [17], we have

$$
\begin{aligned}
v(x) & =Q \sin \left(s(x-r)+\psi_{0}\right) \exp (-\eta x) \\
& -\frac{1}{s} \int_{A}^{x} M(\tau) \exp [-\eta(x-\tau+\Delta(\tau))] \sin s(x-\tau) v(\tau-\Delta(\tau)) d \tau .
\end{aligned}
$$

If $Q=0$, the bound (2.8) is trivial. Suppose $Q>0, x \in\{x: A<x<\infty\} /\{r\}$ and $D_{x_{p} \lambda}=\max _{\left[A, x_{p}\right]}|v(x)|$. For $s>0$ from (2.5) and (2.9), we obtain

$$
\begin{aligned}
D_{x_{p} \lambda} & \leq Q \exp (-\eta A)+\frac{1}{s} D_{x_{p} \lambda} \int_{A}^{x_{p}}|M(\tau)| \exp \left[-\eta\left(x_{p}-\tau\right)\right] d \tau \\
& \leq Q \exp (-\eta A)+\frac{1}{s} D_{x_{p} \lambda} \frac{M_{p}}{\eta}\left(1-\exp \left[-\eta\left(x_{p}-A\right)\right]\right) \\
& \leq Q \exp (-\eta A)+\frac{D_{x_{p} \lambda} M_{p}}{s \eta}
\end{aligned}
$$

and, according to inequality (2.7),

$$
|v(x)|=|u(x)| \exp (-\eta x) \leq \frac{\eta \sqrt{\lambda} Q \exp (-\eta A)}{\eta \sqrt{\lambda}-M_{p}} .
$$

We multiply this expression by $\exp (\eta x)$ and obtain the bound (2.8) for the solution $u_{2}(x)$. Here $B_{02}=\frac{\eta \sqrt{\lambda} Q}{\eta \sqrt{\lambda}-M_{p}}$. The bound for $u_{2}^{\prime}(x)$ can be obtained in a similar way.

Theorem 2.4. In (1.2) and (1.6), let $\sin \alpha=0$, and in (1.1), let $0 \leq M(x) \leq$ $M_{0}<\infty, x \in[0, r) ; 0 \leq M(x) \leq M_{p}<\infty, x \in(r, \infty)$,

$$
\int_{0}^{r}\left(M_{0}-M(\tau)\right) d \tau+\int_{r}^{\infty}\left(M_{p}-M(\tau)\right) d \tau<\infty,
$$

$x-\Delta(x) \geq 0$ for $x \geq 0$. Moreover, assume that $\eta>0, \epsilon>0$ and $C>0$ are the numbers such that

$$
0 \leq \Delta(x) \leq C \exp [-x(\eta+\epsilon)], \quad x \in[0, r) \cup(r, \infty) .
$$

Then all values of $\lambda$ which satisfy the inequality

$$
\eta \sqrt{\lambda}>\max \left\{M_{0}, M_{p}\right\}
$$

are eigenvalues of the boundary-value problem (1.1)-(1.5).

Proof. The proof is similar to that of Theorem 4.2.2 in [17] and Theorem 2 in [18]. 


\section{Theorems on lower bounds for eigenvalues of the prob- lem $(1.1)-(1.5)$}

Lemma 3.1. In (1.4) and (1.6), let

$$
\begin{gathered}
\phi(x) \geq 0, \quad x \in E_{0} \cup E_{1}, \\
\sin \alpha \cos \alpha \leq 0,
\end{gathered}
$$

and in $(1.1)$, let $M(x) \leq 0$ and $\delta>0$ on $[0, r) \cup(r, \infty)$. If $\lambda \leq 0$, then $w(x, \lambda) \neq$ 0 and $w^{\prime}(x, \lambda) \neq 0$ on $(0, r) \cup(r, \infty)$.

Proof. Without loss of generality, we consider the case

$$
\sin \alpha \geq 0 \text { and } \cos \alpha \leq 0
$$

in (3.2). Let $\lambda \leq 0$. From (1.12)-(1.11), we have

$$
\begin{aligned}
& w_{1}^{\prime}(x, 0)=|\cos \alpha|+\int_{0}^{x}|M(\tau)| w_{1}(\tau-\Delta(\tau), 0) d \tau, x \in[0, r), \\
& w_{1}^{\prime}(x, \lambda)= m \sin \alpha \sinh m x+|\cos \alpha| \cosh m x \\
&+\int_{0}^{x}|M(\tau)| \cosh m(x-\tau) w_{1}(\tau-\Delta(\tau), \lambda) d \tau, \\
& m=\sqrt{-\lambda}, x \in[0, r), \\
& w_{2}^{\prime}(x, \lambda)=\frac{m}{\delta} w_{1}(r, \lambda) \sinh m(x-r)+\frac{1}{\delta} w_{1}^{\prime}(r, \lambda) \cosh m(x-r) \\
&+\int_{r}^{x}|M(\tau)| \cosh m(x-\tau) w_{2}(\tau-\Delta(\tau), \lambda) d \tau, \\
& m=\sqrt{-\lambda}, x \in(r, \infty) .
\end{aligned}
$$

Assume $w_{1}(x, \lambda)$ has a zero for $0<x<r$ (if not, from (1.6), (1.9), (3.1), (3.3), and $(3.4), w_{1}(x, \lambda) \neq 0$ and $w_{1}^{\prime}(x, \lambda) \neq 0$ for all $\left.x \in(0, r)\right)$. Let $x_{f}^{\prime}$ be the greatest lower bound of the zeros of $w_{1}(x, \lambda)$. From (1.6), (1.9) and (3.1), it follows that $x_{f}^{\prime}>0, w_{1}(x, \lambda)>0\left(0<x<x_{f}^{\prime}\right)$ and $w_{1}\left(x_{f}^{\prime}, \lambda\right)=0$. According to (3.3), $w_{1}(x-\Delta(x), \lambda) \geq 0$ if $x-\Delta(x)<0$. Thus, $w_{1}(x-\Delta(x), \lambda) \geq 0(0 \leq$ $\left.x \leq x_{f}^{\prime}\right)$, and by (3.4) and (3.5), $w_{1}^{\prime}(x, \lambda)>0\left(0<x \leq x_{f}^{\prime}\right)$. But this contradicts the assumption that $w_{1}\left(x_{f}^{\prime}, \lambda\right)=0$.

Now assume $w_{2}(x, \lambda)$ has a zero for $x>r$ (if not, from (1.7), (1.11), (3.1), (3.3), and (3.6), $w_{2}(x, \lambda) \neq 0$ and $w_{2}^{\prime}(x, \lambda) \neq 0$ for all $\left.x \in(r, \infty)\right)$. Let $x_{f}^{\prime \prime}$ be the greatest lower bound of the zeros of $w_{2}(x, \lambda)$. From (1.7), (1.11), (3.1), (3.3) and (3.6), it follows that $x_{f}^{\prime \prime}>r, w_{2}(x, \lambda)>0\left(r<x<x_{f}^{\prime \prime}\right)$ and $w_{2}\left(x_{f}^{\prime \prime}, \lambda\right)=0$. According to (3.3), $w_{2}(x-\Delta(x), \lambda) \geq 0$ if $x-\Delta(x)<r$. Thus, $w_{2}(x-\Delta(x), \lambda) \geq$ $0\left(r \leq x \leq x_{f}^{\prime \prime}\right)$, and by (1.9), (3.1), (3.3), (3.5), and (3.6), $w_{2}^{\prime}(x, \lambda)>0(r<x \leq$ $\left.x_{f}^{\prime \prime}\right)$. But this contradicts the assumption that $w_{2}\left(x_{f}^{\prime \prime}, \lambda\right)=0$.

Theorem 3.2. Under the hypothesis of Lemma 3.1, the problem (1.1)-(1.5) has no negative eigenvalues. 
Proof. It suffices to consider the case (3.3). Then, by (1.9) and (1.11), it implies that

$$
\begin{aligned}
w_{1}(x, \lambda)= & \sin \alpha \cosh m x+\frac{|\cos \alpha|}{m} \sinh m x \\
& +\frac{1}{m} \int_{0}^{x}|M(\tau)| \sinh m(x-\tau) w_{1}(\tau-\Delta(\tau), \lambda) d \tau, \\
w_{2}(x, \lambda)= & \frac{1}{\delta} w_{1}(r, \lambda) \cosh m(x-r)+\frac{w_{1}^{\prime}(r, \lambda)}{m \delta} \sinh m(x-r) \\
& +\frac{1}{m} \int_{r}^{x}|M(\tau)| \sinh m(x-\tau) w_{2}(\tau-\Delta(\tau), \lambda) d \tau,
\end{aligned}
$$

and the assertion of the theorem follows from Lemma 3.1.

Theorem 3.3. In equation (1.1), suppose that $0 \leq M(x) \leq M_{0}<\infty, x \in$ $[0, r) \cup(r, \infty)$ and that (3.1) and (3.2) hold, with $\phi(x) \leq 1$. Then the boundaryvalue problem (1.1)-(1.5) has no eigenvalues less than $-M_{0}$.

Proof. It suffices to consider the case (3.3). Together with (1.1), we consider the equation without retardation

$$
u^{\prime \prime}(x)+\left(M(x)-M_{0}\right) u(x)=0
$$

and suppose that $z(x)$ is a solution of equation (3.7), which satisfies the conditions

$$
\begin{aligned}
& \left\{\begin{array}{l}
z_{1}(0)=w_{1}(0, \lambda)=\sin \alpha, \\
z_{1}^{\prime}(0)=w_{1}^{\prime}(0, \lambda)=-\cos \alpha,
\end{array}\right. \\
& \left\{\begin{array}{l}
z_{2}(r)=w_{2}(r, \lambda)=\delta^{-1} z_{1}(r), \\
z_{2}^{\prime}(r)=w_{2}^{\prime}(r, \lambda)=\delta^{-1} z_{1}^{\prime}(r) .
\end{array}\right.
\end{aligned}
$$

We will show that if $\lambda<-M_{0}$, then

$$
w(x, \lambda) \geq z(x), \quad x \in[0, r) \cup(r, \infty) .
$$

We will prove it first for the solution $w_{\epsilon}(x, \lambda)$ of (1.1) satisfying the conditions

$$
\left\{\begin{array}{l}
w_{1 \epsilon}(0, \lambda)=\sin \alpha+\epsilon, \quad w_{1 \epsilon}^{\prime}(0, \lambda)=-\cos \alpha+\epsilon, \\
w_{2 \epsilon}(r, \lambda)=\delta^{-1} w_{1 \epsilon}(r, \lambda), \quad w_{2 \epsilon}^{\prime}(r, \lambda)=\delta^{-1} w_{1 \epsilon}^{\prime}(r, \lambda), \\
w_{1 \epsilon}(x-\Delta(x), \lambda)=(\sin \alpha+\epsilon) \phi_{1 \epsilon}(x-\Delta(x)), \quad \text { if } x-\Delta(x)<0 \\
w_{2 \epsilon}(x-\Delta(x), \lambda)=\delta^{-1} w_{1 \epsilon}(r, \lambda) \phi_{2 \epsilon}(x-\Delta(x)), \quad \text { if } x-\Delta(x)<r .
\end{array}\right.
$$

Here $\epsilon>0$ is arbitrary, and

$$
\phi_{\epsilon}(x)= \begin{cases}\phi(x), & \text { if } \phi(x)>\epsilon, \\ \epsilon, & \text { if } \phi(x) \leq \epsilon .\end{cases}
$$

We also show that on $[0, r) \cup(r, \infty)$,

$$
w_{\epsilon}(x, \lambda) \geq z(x) .
$$


Assume the contrary. Let $x_{p}$ be the greatest lower bound of the values of $x$ for which $w_{\epsilon}^{\prime}(x, \lambda)<z^{\prime}(x)$. From (3.7)-(3.9) and (3.11), it follows that $x_{p}>0$. By definition of the point $x_{p}$, we have $w_{\epsilon}^{\prime}\left(x_{p}, \lambda\right)=z^{\prime}\left(x_{p}\right)$ and

$$
w_{\epsilon}^{\prime}(x, \lambda) \geq z^{\prime}(x), \quad w_{\epsilon}(x, \lambda) \geq z(x), \quad 0 \leq x \leq x_{p}<r .
$$

Therefore, $\frac{\partial^{2} w_{\epsilon}\left(x_{p}, \lambda\right)}{\partial x^{2}} \leq \frac{\partial^{2} z\left(x_{p}\right)}{\partial x^{2}}$ and from (1.1) and (3.7),

$$
-\lambda w_{\epsilon}\left(x_{p}, \lambda\right)-M\left(x_{p}\right) w_{\epsilon}\left(x_{p}-\Delta\left(x_{p}\right), \lambda\right) \leq\left(M_{0}-M\left(x_{p}\right)\right) z\left(x_{p}\right) .
$$

Applying Lemma 3.1 to equation (3.7), we find by (3.4) that $z^{\prime}(x) \geq 0$ on $[0, r) \cup$ $(r, \infty)$, and from (3.11) and (3.13),

$$
w_{\epsilon}(x, \lambda) \geq w_{\epsilon}(x-\Delta(x), \lambda) \geq \epsilon^{2}>0, \quad 0 \leq x \leq x_{p}<r .
$$

Then,

$$
\left(-\lambda-M\left(x_{p}\right)\right) w_{\epsilon}\left(x_{p}, \lambda\right) \leq\left(M_{0}-M\left(x_{p}\right)\right) z\left(x_{p}\right) .
$$

Let $M\left(x_{p}\right) \neq M_{0}$ (otherwise inequalities (3.14) and (3.15) are contradictory, and thus (3.12) is proved). Then $-\lambda-M\left(x_{p}\right)>M_{0}-M\left(x_{p}\right)>0$, and from (3.15), $w_{\epsilon}\left(x_{p}, \lambda\right)<z\left(x_{p}\right)$, which contradicts the second inequality in (3.13). Inequality (3.12) is therefore constructed. (3.10) now follows from Theorem 1.3.1 in [17].

If $\cos \alpha \neq 0$ or $M(x) \neq M_{0}$, then applying it to equation (3.7), we obtain $\lim _{x \rightarrow \infty} z(x)=\infty$, and the assertion of the theorem follows from (3.10).

But if $\cos \alpha=0$ and $M(x)=M_{0}$, then $z(x)=1$, and therefore (3.7) cannot be used as a comparison equation.

Suppose that in (1.2) and (1.6), $\cos \alpha=0$ and in (1.1), $M(x)=M_{0}$ (if $M_{0}=$ 0 , the theorem evidently holds) and $\lambda=-\left(M_{0}+\theta\right)(\theta>0)$. The solution of the equation

$$
u^{\prime \prime}(x)-\frac{\theta}{2} u(x)=0
$$

satisfying the initial conditions $u(0)=w(0, \lambda)=1, u^{\prime}(0)=w^{\prime}(0, \lambda)=0$, is given by $u(x)=\cosh \sqrt{\theta / 2} x$. We can now verify that

$$
w(x, \lambda) \geq u(x)=\cosh \sqrt{\frac{\theta}{2}} x, \quad x \in[0, r) \cup(r, \infty),
$$

in a way analogous to the proof of (3.10). Thus the proof is completed.

\section{A Sturm-Liouville problem with discontinuous weight function which contains a spectral parameter in the bound- ary conditions}

In this section, we consider the boundary value problem for the differential equation

$$
u^{\prime \prime}(x)+q(x) u(x-\Delta(x))+\lambda \omega(x) u(x)=0
$$

on $\left[0, \frac{\pi}{2}\right) \cup\left(\frac{\pi}{2}, \pi\right]$ with boundary conditions

$$
u(0)=0
$$




$$
u^{\prime}(\pi)+\lambda u(\pi)=0
$$

and transmission conditions

$$
\begin{aligned}
\gamma_{1} u\left(\frac{\pi}{2}-0\right)-\delta_{1} u\left(\frac{\pi}{2}+0\right) & =0 \\
\gamma_{2} u^{\prime}\left(\frac{\pi}{2}-0\right)-\delta_{2} u^{\prime}\left(\frac{\pi}{2}+0\right) & =0
\end{aligned}
$$

where $\omega(x)$ is a weight function such that $\omega(x)=\omega_{1}^{2}$ if $x \in\left[0, \frac{\pi}{2}\right)$ and $\omega(x)=\omega_{2}^{2}$ if $x \in\left(\frac{\pi}{2}, \pi\right]$, the real-valued function $q(x)$ is continuous on $\left[0, \frac{\pi}{2}\right) \cup\left(\frac{\pi}{2}, \pi\right]$ and it has a finite limit $q\left(\frac{\pi}{2} \pm 0\right)=\lim _{x \rightarrow \frac{\pi}{2} \pm 0} q(x)$, the real-valued function $\Delta(x) \geq 0$ is continuous on $\left[0, \frac{\pi}{2}\right) \cup\left(\frac{\pi}{2}, \pi\right]$ and has a finite limit $\Delta\left(\frac{\pi}{2} \pm 0\right)=\lim _{x \rightarrow \frac{\pi}{2} \pm 0} \Delta(x)$, $x-\Delta(x) \geq 0$, if $x \in\left[0, \frac{\pi}{2}\right) ; x-\Delta(x) \geq \frac{\pi}{2}$, if $x \in\left(\frac{\pi}{2}, \pi\right] ; \lambda$ is a real spectral parameter; $\omega_{1}, \omega_{2}, \gamma_{1}, \gamma_{2}, \delta_{1}, \delta_{2}$ are arbitrary real numbers, and $\omega_{i}>0, \gamma_{i} \neq 0, \delta_{i} \neq$ 0 for $i=1,2$.

Let $\chi_{1}(x, \lambda)$ be a solution of equation (4.1) on $\left[0, \frac{\pi}{2}\right]$ satisfying the initial conditions

$$
\chi_{1}(0, \lambda)=0, \quad \chi_{1}^{\prime}(0, \lambda)=-1 .
$$

The conditions (4.6) define a unique solution of (4.1) on $\left[0, \frac{\pi}{2}\right]$ [17, p. 2].

After defining the above solution, we will define the solution $\chi_{2}(x, \lambda)$ of equation $(4.1)$ on $\left[\frac{\pi}{2}, \pi\right]$ by means of the solution $w_{1}(x, \lambda)$ and using the initial conditions:

$$
\chi_{2}\left(\frac{\pi}{2}, \lambda\right)=\gamma_{1} \delta_{1}^{-1} \chi_{1}\left(\frac{\pi}{2}, \lambda\right), \quad \chi_{2}^{\prime}\left(\frac{\pi}{2}, \lambda\right)=\gamma_{2} \delta_{2}^{-1} \chi_{1}^{\prime}\left(\frac{\pi}{2}, \lambda\right) .
$$

The conditions (4.7) are defined as a unique solution of (4.1) on $\left[\frac{\pi}{2}, \pi\right]$.

Consequently, the function $\chi(x, \lambda)$, defined on $\left[0, \frac{\pi}{2}\right) \cup\left(\frac{\pi}{2}, \pi\right]$ by the equality

$$
\chi(x, \lambda)= \begin{cases}\chi_{1}(x, \lambda), & x \in\left[0, \frac{\pi}{2}\right), \\ \chi_{2}(x, \lambda), & x \in\left(\frac{\pi}{2}, \pi\right],\end{cases}
$$

is the solution of equation (4.1) on $\left[0, \frac{\pi}{2}\right) \cup\left(\frac{\pi}{2}, \pi\right]$, which satisfies one of the boundary conditions and both transmission conditions.

Lemma 4.1. Let $\chi(x, \lambda)$ be a solution of equation (1.1), $\mu=\sqrt{\lambda}$ and $\lambda>0$. Then the following integral equations hold:

$$
\begin{aligned}
\chi_{1}(x, \lambda)= & -\frac{1}{\omega_{1} \mu} \sin \omega_{1} \mu x \\
& -\frac{\omega_{1}}{\mu} \int_{0}^{x} q(\tau) \sin \omega_{1} \mu(x-\tau) \chi_{1}(\tau-\Delta(\tau), \lambda) d \tau \\
\chi_{2}(x, \lambda)= & \frac{\gamma_{1}}{\delta_{1}} \chi_{1}\left(\frac{\pi}{2}, \lambda\right) \cos \mu \omega_{2}\left(x-\frac{\pi}{2}\right)+\frac{\gamma_{2} \chi_{1}^{\prime}\left(\frac{\pi}{2}, \lambda\right)}{\mu \omega_{2} \delta_{2}} \sin \omega_{2} \mu\left(x-\frac{\pi}{2}\right) \\
& -\frac{\omega_{2}}{\mu} \int_{\pi / 2}^{x} q(\tau) \sin \omega_{2} \mu(x-\tau) \chi_{2}(\tau-\Delta(\tau), \lambda) d \tau
\end{aligned}
$$


Proof. To prove this, it is enough to substitute $-\omega_{1}^{2} \mu^{2} \chi_{1}(\tau, \lambda)-\chi_{1}^{\prime \prime}(\tau, \lambda)$ and $-\omega_{2}^{2} \mu^{2} \chi_{2}(\tau, \lambda)-\chi_{2}^{\prime \prime}(\tau, \lambda)$ for $-\omega_{1}^{2} q(\tau) \chi_{1}(\tau-\Delta(\tau), \lambda)$ and $-\omega_{2}^{2} q(\tau) \chi_{2}(\tau-$ $\Delta(\tau), \lambda)$ in the integrals in (4.8) and (4.9), respectively, and integrate by parts twice.

Theorem 4.2. The problem (4.1)-(4.5) can have only simple eigenvalues.

Proof. The proof is similar to that of Theorem 2.1.1 from [17].

The function $\chi(x, \lambda)$, which is defined above, is a nontrivial solution of equation (4.1) satisfying conditions (4.2), (4.4), and (4.5). Putting $\chi(x, \lambda)$ into (4.3), we get the characteristic equation

$$
G(\lambda) \equiv \chi^{\prime}(\pi, \lambda)+\lambda \chi(\pi, \lambda)=0 .
$$

By Theorem 4.2, the set of eigenvalues of the boundary-value problem (4.1)(4.5) coincides with the set of real roots of equation (4.10).

Differentiating (4.8) and (4.9) with respect to $x$, we have

$$
\begin{aligned}
\frac{\partial \chi_{1}(x, \lambda)}{\partial x}= & -\cos \omega_{1} \mu x-\omega_{1}^{2} \int_{0}^{x} q(\tau) \cos \omega_{1} \mu(x-\tau) \chi_{1}(\tau-\Delta(\tau), \lambda) d \tau \\
\frac{\partial \chi_{2}(x, \lambda)}{\partial x}= & -\frac{\omega_{2} \mu \gamma_{1}}{\delta_{1}} \chi_{1}\left(\frac{\pi}{2}, \lambda\right) \sin \omega_{2} \mu\left(x-\frac{\pi}{2}\right) \\
& +\frac{\gamma_{2} \chi_{1}^{\prime}\left(\frac{\pi}{2}, \lambda\right)}{\delta_{2}} \cos \omega_{2} \mu\left(x-\frac{\pi}{2}\right) \\
& -\omega_{2}^{2} \int_{\pi / 2}^{x} q(\tau) \cos \omega_{2} \mu(x-\tau) \chi_{2}(\tau-\Delta(\tau), \lambda) d \tau
\end{aligned}
$$

Let $Q_{1}=\int_{0}^{\pi / 2}|q(\tau)| d \tau$ and $Q_{2}=\int_{\pi / 2}^{\pi} q(\tau) d \tau$.

Let $\lambda \geq v^{2} \max \left\{Q_{1}^{2}, Q_{2}^{2}\right\}$ for $v>1(v \in \mathbb{R})$. Then for the solution $\chi_{1}(x, \lambda)$ of equation (4.8), the following inequality holds:

$$
\left|\chi_{1}(x, \lambda)\right| \leq \frac{1}{\left|v-\omega_{1}\right| \omega_{1}\left|Q_{1}\right|}, \quad x \in\left[0, \frac{\pi}{2}\right] .
$$

Let us denote $L_{v}$ by $L_{v}=\frac{1}{\left|v-\omega_{1}\right| \omega_{1}\left|Q_{1}\right|}$. Then for the solution $\chi_{2}(x, \lambda)$ of equation (4.9), the following inequality holds:

$$
\left|w_{2}(x, \lambda)\right| \leq \frac{\delta_{2} \omega_{2} Q_{1} \gamma_{1} v L_{v}+\delta_{1} \gamma_{2}\left(1+\omega_{1}^{2} Q_{1} L_{v}\right)}{\delta_{1} \delta_{2} \omega_{2} Q_{1}\left(v-\omega_{2}\right)}, \quad x \in\left[\frac{\pi}{2}, \pi\right] .
$$

Theorem 4.3. The problem (4.1)-(4.5) has infinitely many positive eigenvalues.

Proof. From (4.8)-(4.12), we get

$$
-\frac{\omega_{2} \mu \gamma_{1}}{\delta_{1}}\left(-\frac{1}{\omega_{1} \mu} \sin \frac{\omega_{1} \mu \pi}{2}\right.
$$




$$
\begin{aligned}
& \left.-\frac{\omega_{1}}{\mu} \int_{0}^{\frac{\pi}{2}} q(\tau) \sin \omega_{1} \mu\left(\frac{\pi}{2}-\tau\right) \chi_{1}(\tau-\Delta(\tau), \lambda) d \tau\right) \sin \frac{\omega_{2} \mu \pi}{2} \\
& +\frac{\gamma_{2}}{\delta_{2}}\left(-\cos \frac{\omega_{1} \mu \pi}{2}-\omega_{1}^{2} \int_{0}^{\frac{\pi}{2}} q(\tau) \cos \omega_{1} \mu\left(\frac{\pi}{2}-\tau\right) \chi_{1}(\tau-\Delta(\tau), \lambda) d \tau\right) \\
& \times \cos \frac{\omega_{2} \mu \pi}{2}-\omega_{2}^{2} \int_{\pi / 2}^{\pi} q(\tau) \cos \omega_{2} \mu(\pi-\tau) \chi_{2}(\tau-\Delta(\tau), \lambda) d \tau \\
& +\lambda\left(\frac { \gamma _ { 1 } } { \delta _ { 1 } } \left[-\frac{1}{\mu \omega_{1}} \sin \frac{\omega_{1} \mu \pi}{2}\right.\right. \\
& \left.-\frac{w_{1}}{\mu} \int_{0}^{\frac{\pi}{2}} q(\tau) \sin \omega_{1} \mu\left(\frac{\pi}{2}-\tau\right) \chi_{1}(\tau-\Delta(\tau), \lambda) d \tau\right] \cos \frac{\omega_{2} \mu \pi}{2} \\
& +\frac{\gamma_{2}}{\delta_{2} \omega_{2} \mu}\left[-\cos \frac{\omega_{1} \mu \pi}{2}-\omega_{1}^{2} \int_{0}^{\frac{\pi}{2}} q(\tau) \cos \omega_{1} \mu\left(\frac{\pi}{2}-\tau\right) \chi_{1}(\tau-\Delta(\tau), \lambda) d \tau\right] \\
& \left.\times \sin \frac{\omega_{2} \mu \pi}{2}-\frac{\omega_{2}}{\mu} \int_{\frac{\pi}{2}}^{\pi} q(\tau) \sin \omega_{2} \mu(\pi-\tau) \chi_{2}(\tau-\Delta(\tau), \lambda) d \tau\right)=0 .
\end{aligned}
$$

Let $\lambda$ be sufficiently large and $\gamma_{1} \delta_{2} \omega_{2}=\gamma_{2} \delta_{1} \omega_{1}$. Then, by (4.10) and (4.11), equation (4.15) can be written in the form

$$
\mu \sin \frac{\mu \pi\left(\omega_{1}+\omega_{2}\right)}{2}+O(1)=0 .
$$

Obviously, for large $\mu$, equation (4.16) has an infinite set of roots. Thus the theorem is proved.

\section{Asymptotic formulas for eigenvalues and eigenfunctions of the problem (4.1)-(4.5)}

Now we begin to study asymptotic properties of eigenvalues and eigenfunctions. In the following, we will assume that $\mu$ is sufficiently large. From (4.8) and (4.13), we get

$$
\chi_{1}(x, \lambda)=O\left(\frac{1}{\mu}\right), \quad x \in\left[0, \frac{\pi}{2}\right] .
$$

From (4.9), (4.11) and (4.14), we get

$$
\chi_{2}(x, \lambda)=O\left(\frac{1}{\mu}\right), \quad x \in\left[\frac{\pi}{2}, \pi\right] .
$$

The existence and continuity of the derivatives $\frac{\partial \chi_{1}(x, \lambda)}{\partial \mu}$ for $0 \leq x \leq \frac{\pi}{2},|\lambda|<\infty$, and $\frac{\partial \chi_{2}(x, \lambda)}{\partial \mu}$ for $\frac{\pi}{2} \leq x \leq \pi,|\lambda|<\infty$, follow from Theorem 1.4.1 in [17]:

$$
\frac{\partial \chi_{1}(x, \lambda)}{\partial \mu}=O(1), x \in\left[0, \frac{\pi}{2}\right], \quad \text { and } \quad \frac{\partial \chi_{2}(x, \lambda)}{\partial \mu}=O(1), x \in\left[\frac{\pi}{2}, \pi\right] .
$$

Let $N$ be a natural number. We say that the number $\lambda$ is close to the number $\frac{4 N^{2}}{\left(\omega_{1}+\omega_{2}\right)^{2}}$ if $\left|\frac{2 N}{\omega_{1}+\omega_{2}}-\mu\right|<\frac{1}{v^{2}}$. 
Theorem 5.1. Let $N$ be a natural number. For each sufficiently large $N$, there is exactly one eigenvalue of the problem (4.1)-(4.5) that is close to $\frac{4 N^{2}}{\left(\omega_{1}+\omega_{2}\right)^{2}}$.

Proof. We consider the expression which is denoted by $O(1)$ in equation (2.8). If formulas (5.1)-(5.3) are taken into consideration, it can be shown by differentiation with respect to $\mu$ that for large $\mu$ this expression has a bounded derivative. It is obvious that for large $\mu$ the roots of equation (4.16) are close to entire numbers. We show that for large $N$, only one root (4.16) is close to each $\frac{4 N^{2}}{\left(\omega_{1}+\omega_{2}\right)^{2}}$. We consider the function $\theta(\mu)=\mu \sin \frac{\mu \pi\left(\omega_{1}+\omega_{2}\right)}{2}+O(1)$. Its derivative, which has the form $\theta^{\prime}(\mu)=\sin \frac{\mu \pi\left(\omega_{1}+\omega_{2}\right)}{2}+\mu \frac{\pi\left(\omega_{1}+\omega_{2}\right)}{2} \cos \frac{\mu \pi\left(\omega_{1}+\omega_{2}\right)}{2}+O(1)$, does not vanish for $\mu$ close to $N$ for sufficiently large $N$. Thus, by Rolle's theorem, we get the assertion of the theorem.

Let $N$ be sufficiently large. In what follows, we denote by $\lambda_{N}=\mu_{N}^{2}$ the eigenvalue of the problem (4.1)-(4.5) that is close to $\frac{4 N^{2}}{\left(\omega_{1}+\omega_{2}\right)^{2}}$. We set $\mu_{N}=$ $\frac{2 N}{\omega_{1}+\omega_{2}}+\delta_{N}$. From (4.16), it follows that $\delta_{N}=O\left(\frac{1}{N}\right)$. Consequently,

$$
\mu_{N}=\frac{2 N}{\omega_{1}+\omega_{2}}+O\left(\frac{1}{N}\right) .
$$

Formula (5.4) makes it possible to obtain asymptotic expressions for the eigenfunction of the problem (4.1)-(4.5). From (4.8), (4.11), and (5.1), we get

$$
\begin{aligned}
\chi_{1}(x, \lambda) & =-\frac{1}{\omega_{1} \mu} \sin \omega_{1} \mu x+O\left(\frac{1}{\mu^{2}}\right), \\
\frac{\partial \chi_{1}(x, \lambda)}{\partial x} & =-\cos \omega_{1} \mu x+O\left(\frac{1}{\mu}\right) .
\end{aligned}
$$

From (4.9), (4.12), (5.1), and (5.2), we get

$$
\chi_{2}(x, \lambda)=-\frac{\gamma_{1}}{\delta_{1} \omega_{1} \mu} \sin \mu\left(\left(\omega_{1}+\omega_{2}\right) x-\frac{\pi}{2}\right)+O\left(\frac{1}{\mu^{2}}\right) .
$$

By substituting (5.4) into (5.5) and (5.7), we derive that

$$
\begin{aligned}
& u_{1 N}=\chi_{1}\left(x, \lambda_{n}\right)=-\frac{\omega_{1}+\omega_{2}}{2 N \omega_{1}} \sin \frac{2 N \omega_{1} x}{\omega_{1}+\omega_{2}}+O\left(\frac{1}{N^{2}}\right), \\
& u_{2 N}=\chi_{2}\left(x, \lambda_{n}\right)=-\frac{\gamma_{1}\left(\omega_{1}+\omega_{2}\right)}{2 N \delta_{1} \omega_{1}} \sin \left(2 N x-\frac{\pi N}{\omega_{1}+\omega_{2}}\right)+O\left(\frac{1}{N^{2}}\right) .
\end{aligned}
$$

Hence the eigenfunctions $u_{N}(x)$ have the asymptotic representation

$$
u_{N}(x)= \begin{cases}-\frac{\omega_{1}+\omega_{2}}{2 N \omega_{1}} \sin \frac{2 N \omega_{1} x}{\omega_{1}+\omega_{2}}+O\left(\frac{1}{N^{2}}\right), & \text { for } x \in\left[0, \frac{\pi}{2}\right), \\ -\frac{\gamma_{1}\left(\omega_{1}+\omega_{2}\right)}{2 N \delta_{1} \omega_{1}} \sin \left(2 N x-\frac{\pi N}{\omega_{1}+\omega_{2}}\right)+O\left(\frac{1}{N^{2}}\right), & \text { for } x \in\left(\frac{\pi}{2}, \pi\right] .\end{cases}
$$

Under some additional conditions the more exact asymptotic formulas, which depend on the retardation, can be obtained. Let us assume that the following conditions are fulfilled: 
a) the derivatives $q^{\prime}(x)$ and $\Delta^{\prime \prime}(x)$ exist and are bounded on $\left[0, \frac{\pi}{2}\right) \bigcup\left(\frac{\pi}{2}, \pi\right]$ and have finite limits $q^{\prime}\left(\frac{\pi}{2} \pm 0\right)=\lim _{x \rightarrow \frac{\pi}{2} \pm 0} q^{\prime}(x)$ and $\Delta^{\prime \prime}\left(\frac{\pi}{2} \pm 0\right)=\lim _{x \rightarrow \frac{\pi}{2} \pm 0} \Delta^{\prime \prime}(x)$, respectively;

b) $\Delta^{\prime}(x) \leq 1$ on $\left[0, \frac{\pi}{2}\right) \bigcup\left(\frac{\pi}{2}, \pi\right], \Delta(0)=0$ and $\lim _{x \rightarrow \frac{\pi}{2}+0} \Delta(x)=0$.

By using b), we have

$$
x-\Delta(x) \geq 0, \quad \text { for } x \in\left[0, \frac{\pi}{2}\right) \quad \text { and } \quad x-\Delta(x) \geq \frac{\pi}{2}, \quad \text { for } x \in\left(\frac{\pi}{2}, \pi\right] .
$$

From (5.5), (5.7), and (5.8), we have

$$
\begin{aligned}
\chi_{1}(\tau-\Delta(\tau), \lambda) & =-\frac{1}{\omega_{1} \mu} \sin \left(\omega_{1} \mu(\tau-\Delta(\tau))\right)+O\left(\frac{1}{\mu^{2}}\right), \\
\chi_{2}(\tau-\Delta(\tau), \lambda)=-\frac{\gamma_{1}}{\delta_{1} \omega_{1} \mu} \sin \mu\left(\omega_{2}(\tau-\Delta(\tau))+\frac{\left(\omega_{1}-\omega_{2}\right) \pi}{2}\right) & +O\left(\frac{1}{\mu^{2}}\right) .
\end{aligned}
$$

Under conditions a) and $\mathbf{b}$ ) the formulas

$$
O\left(\frac{1}{\mu}\right)= \begin{cases}\int_{0}^{x} q(\tau) \sin \left(\mu \omega_{1}(2 \tau-\Delta(\tau))\right) d \tau, & 0 \leq x \leq \frac{\pi}{2} \\ \int_{0}^{x} q(\tau) \cos \left(\mu \omega_{1}(2 \tau-\Delta(\tau))\right) d \tau, & 0 \leq x \leq \frac{\pi}{2} \\ \int_{\frac{\pi}{2}}^{x} q(\tau) \sin \left(\mu \omega_{2}(2 \tau-\Delta(\tau))\right) d \tau, & \frac{\pi}{2} \leq x \leq \pi \\ \int_{\frac{\pi}{2}}^{x} q(\tau) \cos \left(\mu \omega_{2}(2 \tau-\Delta(\tau))\right) d \tau, & \frac{\pi}{2} \leq x \leq \pi\end{cases}
$$

can be proved by using the same technique as in Lemma 3.3.3 from [17]. Putting these expressions into (4.15), we have

$$
\begin{aligned}
-\frac{\gamma_{1} \omega_{2} \mu}{\delta_{1}}\left[-\frac{1}{\omega_{1} \mu} \sin \frac{\mu \omega_{1} \pi}{2}-\frac{\omega_{1}}{\mu} \int_{0}^{\frac{\pi}{2}} q(\tau) \sin \left(\mu \omega_{1}\left(\frac{\pi}{2}-\tau\right)\right)\right. \\
\left.\times\left(-\frac{1}{\omega_{1} \mu} \sin \left(\omega_{1} \mu(\tau-\Delta(\tau))\right)+O\left(\frac{1}{\mu^{2}}\right)\right) d \tau\right] \sin \frac{\mu \omega_{2} \pi}{2} \\
+\frac{\gamma_{2}}{\delta_{2}}\left[-\cos \frac{\mu \omega_{1} \pi}{2}-\omega_{1}^{2} \int_{0}^{\frac{\pi}{2}} q(\tau) \cos \left(\mu \omega_{1}\left(\frac{\pi}{2}-\tau\right)\right)\right. \\
\left.\quad \times\left(-\frac{1}{\omega_{1} \mu} \sin \left(\omega_{1} \mu(\tau-\Delta(\tau))\right)+O\left(\frac{1}{\mu^{2}}\right)\right) d \tau\right] \cos \frac{\mu \omega_{2} \pi}{2} \\
-\omega_{2}^{2} \int_{\frac{\pi}{2}}^{\pi} q(\tau) \cos \left(\mu \omega_{2}(\pi-\tau)\right) \\
\times\left(-\frac{\gamma_{1}}{\delta_{1} \omega_{1} \mu} \sin \mu\left(\omega_{2}(\tau-\Delta(\tau))+\frac{\left(\omega_{1}-\omega_{2}\right) \pi}{2}\right)+O\left(\frac{1}{\mu^{2}}\right)\right) d \tau \\
+\lambda \frac{\gamma_{1}}{\delta_{1}}\left[-\frac{1}{\mu \omega_{1}} \sin \frac{\mu \omega_{1} \pi}{2}-\frac{\omega_{1}}{\mu} \int_{0}^{\frac{\pi}{2}} q(\tau) \sin \left(\mu \omega_{1}\left(\frac{\pi}{2}-\tau\right)\right)\right.
\end{aligned}
$$




$$
\begin{aligned}
& \left.\times\left(-\frac{1}{\omega_{1} \mu} \sin \left(\omega_{1} \mu(\tau-\Delta(\tau))\right)+O\left(\frac{1}{\mu^{2}}\right)\right) d \tau\right] \cos \frac{\mu \omega_{2} \pi}{2} \\
& +\frac{\gamma_{2}}{\delta_{2} \omega_{2} \mu}\left[-\cos \frac{\mu \omega_{1} \pi}{2}-\omega_{1}^{2} \int_{0}^{\frac{\pi}{2}} q(\tau) \cos \left(\mu \omega_{1}\left(\frac{\pi}{2}-\tau\right)\right)\right. \\
& \left.\times\left(-\frac{1}{\omega_{1} \mu} \sin \left(\omega_{1} \mu(\tau-\Delta(\tau))\right)+O\left(\frac{1}{\mu^{2}}\right)\right) d \tau\right] \sin \frac{\mu \omega_{2} \pi}{2} \\
& -\frac{\omega_{2}}{\mu} \int_{\frac{\pi}{2}}^{\pi} q(\tau) \sin \left(\mu \omega_{2}(\pi-\tau)\right) \\
& \times\left(-\frac{\gamma_{1}}{\delta_{1} \omega_{1} \mu} \sin \mu\left(\omega_{2}(\tau-\Delta(\tau))+\frac{\left(\omega_{1}-\omega_{2}\right) \pi}{2}\right)+O\left(\frac{1}{\mu^{2}}\right)\right) d \tau \\
& =-\frac{\mu \gamma_{1}}{\delta_{1} \omega_{1}} \sin \frac{\mu \pi\left(\omega_{1}+\omega_{2}\right)}{2}-\frac{\gamma_{2}}{\delta_{2}} \cos \frac{\mu \pi\left(\omega_{1}+\omega_{2}\right)}{2} \\
& +\frac{\gamma_{1} \omega_{2} A\left(\frac{\pi}{2}, \lambda\right)}{\delta_{1} \mu} \cos \frac{\mu \pi\left(\omega_{1}+\omega_{2}\right)}{2}-\frac{\gamma_{1} \omega_{2} B\left(\frac{\pi}{2}, \lambda\right)}{\delta_{1} \mu} \sin \frac{\mu \pi\left(\omega_{1}+\omega_{2}\right)}{2} \\
& +\frac{\gamma_{1} B\left(\frac{\pi}{2}, \lambda\right)}{\delta_{1}} \cos \frac{\mu \pi\left(\omega_{1}+\omega_{2}\right)}{2}+\frac{\gamma_{1} A\left(\frac{\pi}{2}, \lambda\right)}{\delta_{1}} \sin \frac{\mu \pi\left(\omega_{1}+\omega_{2}\right)}{2} \\
& -\frac{\omega_{2}^{2} \gamma_{1} C(\pi, \lambda)}{\mu \delta_{1} \omega_{1}} \cos \frac{\mu \pi\left(\omega_{1}+\omega_{2}\right)}{2}+\frac{\omega_{2}^{2} \gamma_{1} D(\pi, \lambda)}{\mu \delta_{1} \omega_{1}} \sin \frac{\mu \pi\left(\omega_{1}+\omega_{2}\right)}{2} \\
& +\frac{\omega_{2} \gamma_{1} D(\pi, \lambda)}{\delta_{1} \omega_{1}} \cos \frac{\mu \pi\left(\omega_{1}+\omega_{2}\right)}{2}+\frac{\omega_{2} \gamma_{1} C(\pi, \lambda)}{\delta_{1} \omega_{1}} \sin \frac{\mu \pi\left(\omega_{1}+\omega_{2}\right)}{2} \\
&
\end{aligned}
$$

Here,

$$
\begin{aligned}
A(x, \lambda) & =\frac{1}{2} \int_{0}^{x} q(\tau) \sin \left(\mu \omega_{1} \Delta(\tau)\right) d \tau, & & x \in\left[0, \frac{\pi}{2}\right] ; \\
B(x, \lambda) & =\frac{1}{2} \int_{0}^{x} q(\tau) \cos \left(\mu \omega_{1} \Delta(\tau)\right) d \tau, & & x \in\left[0, \frac{\pi}{2}\right] ; \\
C(x, \lambda) & =\frac{1}{2} \int_{\frac{\pi}{2}}^{x} q(\tau) \sin \left(\mu \omega_{2} \Delta(\tau)\right) d \tau, & & x \in\left[\frac{\pi}{2}, \pi\right] ; \\
D(x, \lambda) & =\frac{1}{2} \int_{\frac{\pi}{2}}^{x} q(\tau) \cos \left(\mu \omega_{2} \Delta(\tau)\right) d \tau, & & x \in\left[\frac{\pi}{2}, \pi\right] .
\end{aligned}
$$

It is evident that these functions are bounded in the domain of definition for $0<$ $\lambda<\infty$. Thus, from (5.11), we have

$$
\begin{aligned}
\tan \frac{\mu \pi\left(\omega_{1}+\omega_{2}\right)}{2}= & \frac{1}{\mu}\left[1-\frac{\gamma_{1}\left(\omega_{1} A\left(\frac{\pi}{2}, \lambda\right)-\omega_{2} C(\pi, \lambda)\right)}{\mu \delta_{1} \omega_{1}}\right] \\
& \times\left[-\frac{\delta_{1} \omega_{1} \gamma_{2}}{\gamma_{1} \delta_{2}}+\omega_{1} B\left(\frac{\pi}{2}, \lambda\right)+\omega_{2} D(\pi, \lambda)\right]+O\left(\frac{1}{\mu^{3}}\right) .
\end{aligned}
$$

And using $\mu_{N}=\frac{2 N}{\omega_{1}+\omega_{2}}+\delta_{N}$, we have 


$$
\begin{aligned}
\tan ( & \left.\frac{\delta_{N} \pi\left(\omega_{1}+\omega_{2}\right)}{2}\right) \\
= & \frac{\omega_{1}+\omega_{2}}{2 N}\left[1-\frac{\gamma_{1}\left(\omega_{1}+\omega_{2}\right)\left(\omega_{1} A\left(\frac{\pi}{2}, \frac{2 N}{\omega_{1}+\omega_{2}}\right)-\omega_{2} C\left(\pi, \frac{2 N}{\omega_{1}+\omega_{2}}\right)\right)}{2 N \delta_{1} \omega_{1}}\right] \\
& \times\left[-\frac{\delta_{1} \omega_{1} \gamma_{2}}{\gamma_{1} \delta_{2}}+\omega_{1} B\left(\frac{\pi}{2}, \frac{2 N}{\omega_{1}+\omega_{2}}\right)+\omega_{2} D\left(\pi, \frac{2 N}{\omega_{1}+\omega_{2}}\right)\right]+O\left(\frac{1}{n^{3}}\right) .
\end{aligned}
$$

Thus,

$$
\begin{aligned}
\delta_{N}= & \frac{1}{N \pi}\left[1-\frac{\gamma_{1}\left(\omega_{1}+\omega_{2}\right)\left(\omega_{1} A\left(\frac{\pi}{2}, \frac{2 N}{\omega_{1}+\omega_{2}}\right)-\omega_{2} C\left(\pi, \frac{2 N}{\omega_{1}+\omega_{2}}\right)\right)}{2 N \delta_{1} \omega_{1}}\right] \\
& \times\left[-\frac{\delta_{1} \omega_{1} \gamma_{2}}{\gamma_{1} \delta_{2}}+\omega_{1} B\left(\frac{\pi}{2}, \frac{2 N}{\omega_{1}+\omega_{2}}\right)+\omega_{2} D\left(\pi, \frac{2 N}{\omega_{1}+\omega_{2}}\right)\right]+O\left(\frac{1}{n^{3}}\right),
\end{aligned}
$$

and finally,

$$
\begin{aligned}
\mu_{N} & =\frac{2 N}{\omega_{1}+\omega_{2}}+\frac{1}{N \pi}\left[1-\frac{\gamma_{1}\left(\omega_{1}+\omega_{2}\right)\left(\omega_{1} A\left(\frac{\pi}{2}, \frac{2 N}{\omega_{1}+\omega_{2}}\right)-\omega_{2} C\left(\pi, \frac{2 N}{\omega_{1}+\omega_{2}}\right)\right)}{2 N \delta_{1} \omega_{1}}\right] \\
\times & {\left[-\frac{\delta_{1} \omega_{1} \gamma_{2}}{\gamma_{1} \delta_{2}}+\omega_{1} B\left(\frac{\pi}{2}, \frac{2 N}{\omega_{1}+\omega_{2}}\right)+\omega_{2} D\left(\pi, \frac{2 N}{\omega_{1}+\omega_{2}}\right)\right]+O\left(\frac{1}{N^{3}}\right) . }
\end{aligned}
$$

Thus, we have proven the following theorem.

Theorem 5.2. If conditions $\mathbf{a})$ and $\mathbf{b})$ are satisfied, then the positive eigenvalues $\lambda_{N}=\mu_{N}^{2}$ of the problem (4.1)-(4.5) have asymptotic representation (5.12) for $N \rightarrow \infty$.

We now can give a sharper asymptotic formula for the eigenfunctions. From (4.8) and (5.9), we obtain

$$
\begin{aligned}
\chi_{1}(x, \lambda)= & -\frac{1}{\omega_{1} \mu} \sin \left(\omega_{1} \mu x\right) \\
& -\frac{\omega_{1}}{\mu} \int_{0}^{x} q(\tau) \sin \left(\omega_{1} \mu(x-\tau)\right) \\
& \times\left(-\frac{1}{\omega_{1} \mu} \sin \left(\omega_{1} \mu(\tau-\Delta(\tau))\right)+O\left(\frac{1}{\mu^{2}}\right)\right) d \tau \\
= & -\frac{1}{\omega_{1} \mu} \sin \left(\omega_{1} \mu x\right) \\
& +\frac{1}{\mu^{2}}\left[B(x, \lambda) \cos \left(\mu \omega_{1} x\right)+A(x, \lambda) \sin \left(\mu \omega_{1} x\right)\right]+O\left(\frac{1}{\mu^{3}}\right) .
\end{aligned}
$$

Replacing $\mu$ by $\mu_{N}$ and using (5.12), we have

$$
u_{1 N}(x)=\chi_{1}\left(x, \lambda_{N}\right)=\left[-\frac{\omega_{1}+\omega_{2}}{2 N \omega_{1}}+\frac{\left(\omega_{1}+\omega_{2}\right)^{2}}{4 N^{2}} A\left(x, \frac{2 N}{\omega_{1}+\omega_{2}}\right)\right] \sin \left(\frac{2 N \omega_{1} x}{\omega_{1}+\omega_{2}}\right)
$$




$$
\begin{aligned}
& +\left[-\frac{\omega_{1}+\omega_{2}}{2 N^{2} \omega_{1} \pi}\left(-\frac{\delta_{1} \omega_{1} \gamma_{2}}{\gamma_{1} \delta_{2}}+\omega_{1} B\left(\frac{\pi}{2}, \frac{2 N}{\omega_{1}+\omega_{2}}\right)+\omega_{2} D\left(\pi, \frac{2 N}{\omega_{1}+\omega_{2}}\right)\right)\right. \\
& \left.+\frac{\left(\omega_{1}+\omega_{2}\right)^{2}}{4 N^{2}} B\left(x, \frac{2 N}{\omega_{1}+\omega_{2}}\right)\right] \cos \left(\frac{2 N \omega_{1} x}{\omega_{1}+\omega_{2}}\right)+O\left(\frac{1}{N^{3}}\right) .
\end{aligned}
$$

From (4.11) and (5.9), we have

$$
\begin{aligned}
\frac{\partial \chi_{1}(x, \lambda)}{\partial x}= & -\cos \left(\omega_{1} \mu x\right) \\
& +\frac{\omega_{1}}{\mu}\left[-B(x, \lambda) \sin \left(\mu \omega_{1} x\right)+A(x, \lambda) \cos \left(\mu \omega_{1} x\right)\right]+O\left(\frac{1}{\mu^{2}}\right) .
\end{aligned}
$$

From (4.9), (5.10), (5.11), (5.13), and (5.15), we have

$$
\begin{aligned}
\chi_{2}(x, \lambda)= & \frac{\gamma_{1}}{\delta_{1}}\left\{-\frac{1}{\omega_{1} \mu} \sin \left(\frac{\omega_{1} \mu \pi}{2}\right)+\frac{1}{\mu^{2}}\left[B\left(\frac{\pi}{2}, \lambda\right) \cos \left(\frac{\omega_{1} \mu \pi}{2}\right)\right.\right. \\
& \left.\left.+A\left(\frac{\pi}{2}, \lambda\right) \sin \left(\frac{\omega_{1} \mu \pi}{2}\right)\right]+O\left(\frac{1}{\mu^{3}}\right)\right\} \cos \omega_{2} \mu\left(x-\frac{\pi}{2}\right) \\
& +\frac{\gamma_{2}}{\mu \omega_{2} \delta_{2}}\left\{-\cos \left(\frac{\omega_{1} \mu \pi}{2}\right)+\frac{\omega_{1}}{\mu}\left[-B\left(\frac{\pi}{2}, \lambda\right) \sin \left(\frac{\omega_{1} \mu \pi}{2}\right)\right.\right. \\
& \left.\left.+A\left(\frac{\pi}{2}, \lambda\right) \cos \left(\frac{\omega_{1} \mu \pi}{2}\right)\right]+O\left(\frac{1}{\mu^{2}}\right)\right\} \sin \omega_{2} \mu\left(x-\frac{\pi}{2}\right) \\
& -\frac{\omega_{2}}{\mu} \int_{\frac{\pi}{2}}^{x} q(\tau) \sin \omega_{2} \mu(x-\tau) \\
& \times\left\{\frac{-\gamma_{1}}{\delta_{1} \omega_{1} \mu} \sin \mu\left(\omega_{2}(\tau-\Delta(\tau))+\frac{\left(\omega_{1}-\omega_{2}\right) \pi}{2}\right)+O\left(\frac{1}{\mu^{2}}\right)\right\} d \tau \\
= & \left.-\frac{\gamma_{1}}{\delta_{1} \omega_{1} \mu}+\frac{\gamma_{1}}{\delta_{1} \omega_{1} \mu^{2}}\left(\omega_{1} A\left(\frac{\pi}{2}, \lambda\right)+\omega_{2} C(x, \lambda)\right)\right] \\
& \times \sin \mu\left(\omega_{2} x+\frac{\pi\left(\omega_{1}-\omega_{2}\right)}{2}\right) \\
& +\left[\frac{\gamma_{1}}{\delta_{1} \omega_{1} \mu^{2}}\left(\omega_{1} B\left(\frac{\pi}{2}, \lambda\right)+\omega_{2} D(x, \lambda)\right)\right] \cos \mu\left(\omega_{2} x+\frac{\pi\left(\omega_{1}-\omega_{2}\right)}{2}\right) \\
& +O\left(\frac{1}{\mu^{3}}\right) .
\end{aligned}
$$

Now, replacing $\mu$ by $\mu_{N}$ and using (5.12), we have

$$
\begin{aligned}
u_{2 N}(x)= & \chi_{2}\left(x, \lambda_{N}\right)=\left[-\frac{\gamma_{1}\left(\omega_{1}+\omega_{2}\right)}{2 N \delta_{1} \omega_{1}}+\frac{\left(\omega_{1}+\omega_{2}\right)^{2} \gamma_{1}}{4 N^{2} \delta_{1} \omega_{1}}\left(\omega_{1} A\left(\frac{\pi}{2}, \frac{2 N}{\omega_{1}+\omega_{2}}\right)\right.\right. \\
& \left.\left.+\omega_{2} C\left(x, \frac{2 N}{\omega_{1}+\omega_{2}}\right)\right)\right] \sin \left(\frac{2 N \omega_{2} x+N\left(\omega_{1}-\omega_{2}\right) \pi}{\omega_{1}+\omega_{2}}\right) \\
& +\left[( \frac { 1 } { N \pi } ) ( \omega _ { 2 } x + \frac { ( \omega _ { 1 } - \omega _ { 2 } ) \pi } { 2 } ) \left(-\frac{\delta_{1} \omega_{1} \gamma_{2}}{\gamma_{1} \delta_{2}}+\omega_{1} B\left(\frac{\pi}{2}, \frac{2 N}{\omega_{1}+\omega_{2}}\right)\right.\right.
\end{aligned}
$$




$$
\begin{aligned}
& \left.+\omega_{2} D\left(\pi, \frac{2 N}{\omega_{1}+\omega_{2}}\right)\right)\left(-\frac{\gamma_{1}\left(\omega_{1}+\omega_{2}\right)}{2 N \delta_{1} \omega_{1}}\right) \\
& \left.+\frac{\left(\omega_{1}+\omega_{2}\right)^{2} \gamma_{1}}{4 N^{2} \delta_{1} \omega_{1}}\left(\omega_{1} B\left(\frac{\pi}{2}, \frac{2 N}{\omega_{1}+\omega_{2}}\right)+\omega_{2} D\left(x, \frac{2 N}{\omega_{1}+\omega_{2}}\right)\right)\right] \\
& \times \cos \left(\frac{2 N \omega_{2} x+N\left(\omega_{1}-\omega_{2}\right) \pi}{\omega_{1}+\omega_{2}}\right)+O\left(\frac{1}{N^{3}}\right) .
\end{aligned}
$$

Thus, we have proven the following theorem.

Theorem 5.3. If conditions $\mathbf{a})$ and $\mathbf{b}$ ) are satisfied, then the eigenfunctions $u_{N}(x)$ of the problem (4.1)-(4.5) have the following asymptotic representation for $N \rightarrow \infty$ :

$$
u_{N}(x)= \begin{cases}u_{1 N}(x) & \text { for } x \in\left[0, \frac{\pi}{2}\right), \\ u_{2 N}(x) & \text { for } x \in\left(\frac{\pi}{2}, \pi\right],\end{cases}
$$

where $u_{1 N}(x)$ and $u_{2 N}(x)$ are defined as in (5.14) and (5.16), respectively.

\section{Bounds for the distance between eigenvalues}

Let us define

$$
\chi_{0}=\left\{\begin{array}{l}
\min \left\{\beta_{0}^{2} Q_{\pi}^{2}, \gamma_{0}^{2} Q_{0}^{2} \pi^{2}\right\}, \\
\gamma_{0}^{2} Q_{\pi}^{2}, \text { if } x|q(x)| \geqslant \int_{0}^{x} q(t) d t, \quad 0 \leq x \leq \pi,
\end{array}\right.
$$

where $\beta_{0}$ and $\gamma_{0}$ are the unique real roots of the equations

$$
\beta=(\sqrt{2+\sqrt{2}}+\sqrt{2}) e^{1 / \beta} \text { and } \gamma=\frac{\sqrt{2}}{4}(\sqrt{9+4 \sqrt{2}}+3) e^{1 / \gamma}
$$

respectively;

$$
Q_{\pi}=\int_{0}^{\pi}|q(x)| d x \quad \text { and } \quad Q_{0}=\max _{[0, \pi]}|q(x)| .
$$

Assume that $\lambda \geqq \chi_{0}$ and let $\lambda_{N}, \lambda_{N+1}, \ldots, \lambda_{N+p}, \ldots$ be the eigenvalues of the problem (4.1)-(4.5) listed in increasing order, $N$ be the number of zeros on the set $(0, \pi / 2) \cup(\pi / 2, \pi)$ of the eigenfunctions corresponding to the eigenvalue $\lambda_{N}$. In what follows, the eigenvalues with an odd index will be called odd, and those with an even index will be called even.

Now we will state the following theorem which can be proven easily using the same method as in [17].

Theorem 6.1 (Asymptotic Oscillation Theorem). The eigenvalues of the problem (4.1)-(4.5) form an unbounded increasing sequence $\lambda_{N}, \lambda_{N+1}, \ldots, \lambda_{N+p}$, ..., in the region $\lambda \geqq \chi_{0}$. Moreover, the eigenfunction corresponding to the eigenvalue $\lambda_{N+p}$ has exactly $N+p$ zeros on the set $(0, \pi / 2) \cup(\pi / 2, \pi)$, where $N$ is the number of zeros of the eigenfunction corresponding to the first eigenvalue $\lambda_{N}$ of the sequence. 
Lemma 6.2. Suppose that $\lambda \geqq \chi_{0}$ in (4.1) and that $\lambda^{\prime}$ is an eigenvalue of the problem (4.1)-(4.5). Then $\sqrt{\lambda^{\prime}}=\mu^{\prime}=\frac{2 n^{\prime}}{\omega_{1}+\omega_{2}}+\delta_{n^{\prime}}$, where $n^{\prime}$ is an integer, and $\left|\delta_{n^{\prime}}\right| \leq \frac{1}{\omega_{1}+\omega_{2}}$. Moreover, if $\lambda^{\prime}$ is an odd eigenvalue, then $n^{\prime}$ is even; for an even eigenvalue, $n^{\prime}$ is odd.

Proof. Suppose that $\lambda^{\prime}$ is an odd eigenvalue of the problem (4.1)-(4.5) and that

$$
\sqrt{\lambda^{\prime}}=\mu^{\prime}=\frac{2 n^{\prime}}{\omega_{1}+\omega_{2}}+\delta_{n^{\prime}},
$$

where $n^{\prime}$ is an integer, and

$$
\left|\delta_{n^{\prime}}\right| \leq \frac{1}{\omega_{1}+\omega_{2}} .
$$

Differentiating (4.9) with respect to $x$ and evaluating its value at $x=\pi$, we obtain

$$
\left|\cos \frac{\mu^{\prime} \pi\left(\omega_{1}+\omega_{2}\right)}{2}\right|>\frac{\sqrt{2}}{2} .
$$

However, if $\lambda^{\prime} \geqq \chi_{0}$, from (4.6) and Lemma 2.3.6 in [17] it follows that

$$
\frac{1}{\mu^{\prime}}\left|\int_{\pi / 2}^{\pi} q(\tau) \cos \left(\mu^{\prime} \omega_{2}(\pi-\tau)\right) \chi_{2}\left(\tau-\Delta(\tau), \lambda^{\prime}\right) d \tau\right|<\frac{\sqrt{2}}{2}
$$

yields, and it follows from (6.3) and (6.4) that the sign of the derivative coincides with the sign of $\cos \frac{\mu^{\prime} \pi\left(\omega_{1}+\omega_{2}\right)}{2}$. From Theorem 6.1 and Lemma 2.3.3 in [17], we obtain $\frac{\partial \chi\left(\pi, \lambda^{\prime}\right)}{\partial x}>0$. Therefore we get

$$
\cos \frac{\mu^{\prime} \pi\left(\omega_{1}+\omega_{2}\right)}{2}>0 .
$$

From (6.1), it now follows that

$$
\begin{aligned}
\cos \frac{\mu^{\prime} \pi\left(\omega_{1}+\omega_{2}\right)}{2} & =\cos \left(\left(\frac{2 n^{\prime}}{\omega_{1}+\omega_{2}}+\delta_{n^{\prime}}\right) \frac{\pi\left(\omega_{1}+\omega_{2}\right)}{2}\right) \\
& =\cos n^{\prime} \pi \cos \frac{\delta_{n^{\prime}} \pi\left(\omega_{1}+\omega_{2}\right)}{2} .
\end{aligned}
$$

If the equality sign holds in (6.2), then $\cos \frac{\delta_{n^{\prime}} \pi\left(\omega_{1}+\omega_{2}\right)}{2}=0$, and therefore $\cos \frac{\mu^{\prime} \pi\left(\omega_{1}+\omega_{2}\right)}{2}=0$, which contradicts (6.5). The integer $n^{\prime}$ is defined uniquely and $\left|\frac{\delta_{n^{\prime}} \pi\left(\omega_{1}+\omega_{2}\right)}{2}\right|<\pi / 2$. Then $\cos \frac{\delta_{n^{\prime}} \pi\left(\omega_{1}+\omega_{2}\right)}{2}>0$ and, from (6.5), $\cos n^{\prime} \pi>0$. Thus the proof is completed.

Theorem 6.3. Let $\lambda^{\prime}=\mu_{1}^{2}, \lambda^{\prime \prime}=\mu_{2}^{2}, \lambda^{\prime \prime \prime}=\mu_{3}^{2}\left(\lambda^{\prime \prime \prime}>\lambda^{\prime \prime}>\lambda^{\prime} \geqq \chi_{0}\right)$ be three successive eigenvalues of the problem (4.1)-(4.5). Then

$$
\begin{aligned}
\mu_{2}-\mu_{1} & <\frac{4}{\omega_{1}+\omega_{2}}, \\
\frac{2}{\omega_{1}+\omega_{2}}<\mu_{3}-\mu_{1} & <\frac{6}{\omega_{1}+\omega_{2}}, \\
\mu_{3}-\mu_{2} & <\frac{4}{\omega_{1}+\omega_{2}} .
\end{aligned}
$$


Proof. By Lemma 6.2, $\mu_{3}=\frac{2 n_{3}}{\omega_{1}+\omega_{2}}+\delta_{n_{3}}$ and $\mu_{1}=\frac{2 n_{1}}{\omega_{1}+\omega_{2}}+\delta_{n_{1}}$, with $n_{3}-$ $n_{1} \geq 2$ and $\left|\delta_{n_{1}}\right|<\frac{1}{\omega_{1}+\omega_{2}},\left|\delta_{n_{3}}\right|<\frac{1}{\omega_{1}+\omega_{2}}$. Therefore,

$$
\mu_{3}-\mu_{1} \geq \frac{2\left(n_{3}-n_{1}\right)}{\omega_{1}+\omega_{2}}-\left(\left|\delta_{n_{3}}\right|+\left|\delta_{n_{1}}\right|\right)>\frac{2}{\omega_{1}+\omega_{2}} .
$$

The inequalities (6.6), (6.8), and the second inequality in (6.7) can be proved by using the same method as in the proof of Theorem 3.6.1 from [17].

\section{References}

[1] Z. Akdoğan, M. Demirci, and O.Sh. Mukhtarov, Green function of discontinuous boundary-value problem with transmission conditions, Math. Methods Appl. Sci. 30 (2007), 1719-1738.

[2] J. Ao, J. Sun, M. Zhang, The finite spectrum of Sturm-Liouville problems with transmission conditions, Appl. Math. Comput. 218 (2011), 1166-1173.

[3] A. Bayramov, S. Öztürk Uslu, and S. Kizilbudak Caliṣkan, Computation of eigenvalues and eigenfunctions of a discontinuous boundary value problem with retarded argument, Appl. Math. Comp. 191 (2007), 592-600.

[4] A. Bayramov and E. Şen, On a Sturm-Liouville type problem with retarded argument, Math. Methods Appl. Sci. 36 (2013), 39-48.

[5] R. Bellman and K.L. Cook, Differential-Difference Equations, Academic Press, New York-London, 1963.

[6] P.A. Binding, P.J. Browne, and K. Seddighi, Sturm-Liouville problems with eigenparameter dependent boundary conditions, Proc. Edinb. Math. Soc. (2) 37 (1994), $57-72$.

[7] A. Campbell and S.A. Nazarov, Asymptotics of eigenvalues of a plate with small clamped zone, Positivity 5 (2001), 275-295.

[8] C.T. Fulton, Two-point boundary value problems with eigenvalue parameter contained in the boundary conditions, Proc. Roy. Soc. Edinburgh Sect. A 77 (1977), 293-308.

[9] M. Jabloński and K. Twardowska, On boundary value problems for differential equations with a retarded argument, Univ. Iagel. Acta Math. 26 (1987), 29-36.

[10] M. Kadakal and O. Sh. Mukhtarov, Sturm-Liouville problems with discontinuities at two points, Comput. Math. Appl. 54 (2007), 1367-1379.

[11] B.M. Levitan, Expansion in Characteristic Functions of Differential Equations of the Second Order, Gosudarstv. Izdat. Tehn.-Teor. Lit., Moscow-Leningrad, 1950 (Russian).

[12] Kh.R. Mamedov, On boundary value problem with parameter in boundary conditions, Spectral Theory of Operator and Its Applications, XI, Izdat. "Èlm", Baku, 1997, 117-121 (Russian).

[13] Kh.R. Mamedov and N. Palamut, On a direct problem of scattering theory for a class of Sturm-Liouville operator with discontinuous coefficient, Proc. Jangjeon Math. Soc. 12 (2009), 243-251. 
[14] V.A. Marchenko, Sturm-Liouville operators and applications (Revised ed.), AMS Chelsea Publishing, Providence, RI, 2011.

[15] A.D. Miškis, Linear Differential Equations with Retarded Argument, Gosudarstv. Izdat. Tehn.-Teor. Lit., Moscow-Leningrad, 1951 (Russian).

[16] S.B. Norkin, On boundary problem of Sturm-Liouville type for second-order differential equation with retarded argument, Izv. Vysš. Učebn. Zaved. Matematika, 6 (1958), 203-214 (Russian).

[17] S.B. Norkin, Differential equations of the second order with retarded argument. Some problems of the theory of vibrations of systems with retardation, Translations of Mathematical Monographs, 31, Amer. Math. Soc., Providence, RI, 1972.

[18] Z.I. Rehlickiiu, Test for boundedness of the solutions of linear differential equations with several lags of the argument, Dokl. Akad. Nauk SSSR 125 (1959), 46-47 (Russian).

[19] E. Şen and A. Bayramov, Calculation of eigenvalues and eigenfunctions of a discontinuous boundary value problem with retarded argument which contains a spectral parameter in the boundary condition, Math. Comput. Modelling 54 (2011), 30903097.

[20] E. Şen and A. Bayramov, Asymptotic formulations of the eigenvalues and eigenfunctions for a boundary value problem, Math. Methods Appl. Sci. 36 (2013), 1512-1519.

[21] E. Şen, J.J. Seo, and S. Araci, Asymptotic behaviour of eigenvalues and eigenfunctions of a Sturm-Liouville problem with retarded argument, J. Appl. Math. 2013, Art. ID 306917, 8 pp.

[22] E.C. Titchmarsh, Eigenfunctions Expansion Associated with Second-Order Differential Equations. Part I, Clarendon Press, Oxford, 1962.

[23] E. Tunç and O.Sh. Mukhtarov, Fundamental solutions and eigenvalues of one boundary-value problem with transmission conditions, Appl. Math. Comput. 157 (2004), 347-355.

[24] J. Walter, Regular eigenvalue problems with eigenvalue parameter in the boundary conditions, Math. Z. 133 (1973), 301-312.

Received July 14, 2016, revised June 6, 2017.

Erdoğan Şen,

Namik Kemal University, Department of Mathematics, Faculty of Arts and Science, Tekirdă̆, 59030, Turkey,

E-mail: erdogan.math@gmail.com

\section{Спектральний аналіз розривних задач Штурма-Ліувілля із запізненням аргументу Erdoğan Şen}

У даній статті ми маємо справу із спектральними властивостями розривних задач типу Штурма-Ліувілля із запізненням аргументу. Ми розширюємо і узагальнюємо деякі підходи і результати класичних регулярних і розривних задач Штурма-Ліувілля. Спочатку ми вивчаємо 
спектральні властивості задачі Штурма-Ліувілля на півосі й отримуємо нижні оцінки для власних значень задачі. Потім ми вивчаємо спектральні властивості задачі Штурма-Ліувілля з розривною ваговою функцією, яка містить спектральний параметр в крайових умовах. Ми також отримуємо асимптотичні формули для власних значень і власних функцій задачі та межі відстані між власними значеннями.

Ключові слова: диференціальне рівняння із запізненням аргументу, власний параметр, умови передачі, асимптотика власних значень, межі власних значень. 